\title{
Cosmological parameter estimation in the quintessence paradigm
}

\author{
M. Douspis ${ }^{1,2}$, A. Riazuelo ${ }^{3, \star}$, Y. Zolnierowski ${ }^{1,4,5, \star \star}$, and A. Blanchard ${ }^{1, \star \star \star}$ \\ ${ }^{1}$ Laboratoire d'Astrophysique de l'Observatoire Midi-Pyrénées, 14 avenue E. Belin, 31400 Toulouse, France \\ 2 Nuclear and Astrophysics Laboratory, Keble Road, Oxford, OX1 3RH, UK \\ 3 Service de Physique Théorique, CEA/DSM/SPhT, Unité de recherche associée au CNRS, CEA/Saclay, \\ 91191 Gif-sur-Yvette Cedex, France \\ ${ }^{4}$ L.A.P.P., IN2P3-CNRS, BP 110, 74941 Annecy-le-Vieux Cedex, France \\ 5 Université de Savoie, BP 1104, 73011 Chambéry Cedex, France
}

Received 6 December 2002 / Accepted 21 March 2003

\begin{abstract}
We present cosmological parameter constraints on flat cosmologies dominated by dark energy using various cosmological data including the recent Archeops angular power spectrum measurements. A likelihood analysis of the existing Cosmic Microwave Background data shows that in the absence of further prior, dark energy is not required. This comes from the fact that degeneracies exist among the various cosmological parameters constrained by the Cosmic Microwave Background. We found that there is a degeneracy in a combination of the Hubble parameter $H_{0}$ and of the dark energy equation of state parameter $w_{\mathrm{Q}}$, but that $w_{\mathrm{Q}}$ is not correlated with the primordial index $n$ of scalar fluctuations and the baryon content $\Omega_{\mathrm{b}} h^{2}$. The preferred primordial index is $n=0.95 \pm 0.05(68 \%)$ and baryon content $\Omega_{\mathrm{b}} h^{2}=0.021 \pm 0.003$. Adding constraints on the amplitude of matter fluctuations on small scales, $\sigma_{8}$, obtained from clusters abundance or weak lensing data may allow degenaracies to be broken, although present-day systematic uncertainties do not allow for firm conclusions as yet. Further addition of the Hubble Space Telescope measurements of the local distance scale and of the high redshift supernovae data allow one to obtain tight constraints. When these constraints are combined we find that the amount of dark energy is $0.7_{-0.07}^{+0.10}(95 \%$ C.L.) and that its equation of state is very close to those of the vacuum: $w_{\mathrm{Q}}<-0.75$ ( $>95 \%$ C.L.). In no case do we find that quintessence is prefered over the classical cosmological constant, although robust data on $\sigma_{8}$ might rapidly shed light on this important issue.
\end{abstract}

Key words. cosmology: cosmic microwave background - cosmology: cosmological parameters

\section{Introduction}

The determination of cosmological parameters has always been a central question in cosmology. In this respect the measurements of the Cosmological Microwave Background (CMB) anisotropies on degree angular scales has brought one of the most spectacular results in the field: the flatness of the spatial geometry of the universe, implying that its density is close to the critical density. Although evidence for the existence of nonbaryonic dark matter has strongly gained in robustness during the last twenty years, observations clearly favor a relatively low matter content somewhere between 20 and $50 \%$ of the critical density, thus indicating that the dominant form of the density of the universe is in an unclustered form. Furthermore, the observations of distant supernovae, at cosmological distances, provide direct evidence for an accelerating universe, which is

Send offprint requests to: $\mathrm{M}$. Douspis,

e-mail: douspis@astro.ox.ac.uk

* e-mail: riazuelo@spht.saclay.cea.fr

$\star \star$ e-mail: zolniero@ast.obs-mip.fr

$\star \star \star$ e-mail: alain.blanchard@ast.obs-mip.fr naturally explained by the gravitational domination of a component with a relatively large negative pressure, $P_{\mathrm{Q}}=w_{\mathrm{Q}} \rho_{\mathrm{Q}}$ with $w_{\mathrm{Q}}<-1 / 3$. The cosmological constant $\Lambda$ (for which $w_{\Lambda}=-1$ ) is historically the first possibility which was introduced and satisfies this requirement. However, the presence of a non-zero cosmological constant is a huge problem in physics: (i) quantum field theory predicts that $\Lambda$ should be the sum of a number of enormous contributions, so in order to avoid a cosmological catastrophe, it is usually assumed that a yet unknown mechanism produces a cancelation between all these contributions; (ii) it is difficult to think of a mechanism which puts $\Lambda$ to 0 exactly, but it is even more difficult to find a mechanism which gives $\rho_{\Lambda} \sim \rho_{\mathrm{c}} \sim 10^{-122} \rho_{\mathrm{Pl}}$, as the supernovae observations suggest. (Where $\rho_{\mathrm{Pl}}$ and $\rho_{\mathrm{c}}$ are the Planck energy density and the critical density today respectively.) For this reason the concept of quintessence, a scalar field with negative pressure, has recently been proposed as a possible alternative to a cosmological constant.

In this paper we briefly describe the quintessence paradigm and its effect on some observable quantities. We then summarise the different sets of data and methods used to constrain 
cosmological parameters. We then conclude with our results on quintessence and cosmological parameters.

\section{Quintessence}

The idea of quintessence was proposed in order to allow for the presence of a non-zero dark energy - as suggested by observations - without being confronted by the dramatic finetuning problem of the cosmological constant. Indeed, one still assumes that an unknown mechanism puts the bare cosmological constant is zero, and the smallness of the dark energy has a dynamical origin, coming from a scalar field $\phi$ which has not yet reached the minimum of its potential $V(\phi)$.

A large number of quintessence models were already considered in the literature. Historically, the first proposed quintessence model had an inverse power law potential, $V(\phi)=$ $M^{4+\alpha} / \phi^{\alpha}$ (Ratra \& Peebles 1988; Wetterich 1988; Caldwell et al. 1998), where the exponent $\alpha$ is positive and $M$ is an energy scale fixed so that the scalar field has the correct energy density today. This unusual shape, which can have some motivations from particle physics (Binétruy 1999, 2000), insures that regardless of the initial conditions, the field will reach a so-called "tracking regime" (Steinhardt et al. 1999), in which its pressure and energy density tend to a constant ratio given by $P_{\mathrm{Q}} / \rho_{\mathrm{Q}} \equiv w_{\mathrm{Q}}=\left(\alpha w_{\mathrm{B}}-2\right) /(\alpha+2)$, where $w_{\mathrm{B}}$ is the pressure to energy density ratio of the other background matter fluids (photons, neutrinos, baryons, and cold dark matter). Moreover, since $w_{\mathrm{Q}}<w_{\mathrm{B}}$ in this regime, the quintessence energy density decreases more slowly than that of the background fluids and ultimately the quintessence field will become dominant. When this occurs, i.e., when its density parameter reaches $\Omega_{Q} \gtrsim 0.5$, the field slows down in its potential, and asymptotically reaches $w_{\mathrm{Q}}=-1$ (Steinhardt et al. 1999). The rate at which one goes from the tracking regime to the cosmological regime is usually quite slow, so that unless $w_{\mathrm{Q}}$ is already close to -1 in the tracking regime, it will still be significantly different from -1 when $\Omega_{\mathrm{Q}} \sim 0.7$. For example, if $\alpha=6$, in which case $w_{\mathrm{Q}}=-0.25$ in the tracking regime during the matter-dominated era, one has $w_{\mathrm{Q}}=-0.4$ today if $\Omega_{\mathrm{Q}}=0.7$. This feature leaves open the hope of distinguishing a quintessence field from a cosmological constant.

The effect of a quintessence field on CMB anisotropies is twofold (Brax et al. 2000). First, when the quintessence field becomes dominant, it modifies the expansion rate of the universe. This translates into a modification of the angular distance vs. redshift relation, and hence a shift in the peak structure of the CMB anisotropies power spectrum, the $C_{\ell}$ 's, for $\ell \gtrsim 100$. Second, the gravitational potentials decay at late time as the universe is no longer matter-dominated. This produces a so-called integrated Sachs-Wolfe effect and modifies the $C_{\ell}$ spectrum at low multipoles $(\ell \lesssim 20)$ as a consequence of the fact that photons exchange energy with time-varying gravitational potentials. Both of these effects are also present with a cosmological constant, but they differ quantitatively with a quintessence field: the shift in the peak position is smaller, whereas the integrated Sachs-Wolfe effect can be very different (Caldwell et al. 1998).
Most of the CMB experiments do not cover a large fraction of the sky. On the contrary, the new Archeops data (Benoît et al. 2002; Benoît et al. 2003a) are extremely precise around $\ell \sim 200$ improving by a factor of two the precision measurements on the location of the first Doppler peak (Benoit et al. 2003b), whereas at larger angular scales (low $\ell$ ) the COBE data are limited by a large cosmic variance (Tegmark 1996). Therefore we can hope to be able to contrain the quintessence parameters through their influence on the position of the first Doppler peak rather than through the integrated Sachs-Wolfe effect. It is well-known that the position of the first peak is primarily sensitive to the curvature but also to several other cosmological parameters. It is therefore important to investigate a wide space of parameters in order to constraint the possible existence of quintessence in a robust way.

Finally, let us add that quintessence also modifies significantly the matter power spectrum: as for the cosmological constant, matter fluctuations stop growing at the onset of quintessence domination. This has an influence on the normalization of the matter power spectrum on small scales, $\sigma_{8}$. This effect of $w_{\mathrm{Q}}$ can be understood as follows: as long as $w_{\mathrm{Q}}$ is not too close to 0 , one can roughly consider that the universe has experienced two distinct epochs since recombination: a first (usual) one where it was matter-dominated, and a second one where it is quintessence-dominated. The transition occurs when $\Omega_{\text {mat }}=\Omega_{\mathrm{Q}}=0.5$. If we suppose that $\Omega_{\mathrm{Q}}=0.7$ today, then the transition epoch occured at a redshift of

$z_{\mathrm{Q}}^{\mathrm{mat}}=\left(\frac{\Omega_{\mathrm{Q}}}{\Omega_{\mathrm{mat}}}\right)^{\frac{-1}{3 w_{\mathrm{Q}}}}-1$.

For $w_{\mathrm{Q}}=-1$ (cosmological constant case), one has $z_{\Lambda}^{\text {mat }}=0.33$, whereas for $w_{\mathrm{Q}}=-0.2$, this gives $z_{\mathrm{Q}}^{\mathrm{mat}}=3$.1. If we suppose that perturbations grow as the scale factor before the transition and stop growing immediately afterward, then this corresponds to a reduction of

$R=\left(\frac{1+z_{\Lambda}^{\mathrm{mat}}}{1+z_{\mathrm{Q}}^{\mathrm{mat}}}\right)^{2}=\left(\frac{\Omega_{\mathrm{Q}}}{\Omega_{\mathrm{mat}}}\right)^{\frac{2}{3}\left(1+\frac{1}{w_{\mathrm{Q}}}\right)}$,

in the matter power spectrum as compared to the cosmological constant case. In practice, several factors modify this amount, such as the fact the the matter perturbation still grow (although slowly) in the quintessence-dominated era, but the above analysis illustrates the dramatic change that quintessence can trigger in the matter power spectrum (see also Benabed \& Bernardeau 2001). When $w_{\mathrm{Q}}=0$, the situation is even more dramatic as matter never dominates. In order to understand the value of $\sigma_{8}$, one has to compute the growth of dark matter perturbations when there is a mixture of matter and quintessence with identical equation of state. Neglecting the quintessence fluctuations (which are never subject to any instability), the Jeans equation is rewritten as

$\ddot{\delta}+\mathcal{H} \dot{\delta}-\frac{3}{2} \mathcal{H}^{2} \Omega_{\mathrm{mat}} \delta=0$,

where $\delta$ is the matter perturbation density constrast, an overdot denotes a derivative with respect to conformal time (defined by $a \mathrm{~d} \eta=\mathrm{d} t$, where $a$ is the scale factor and $t$ is the cosmic 


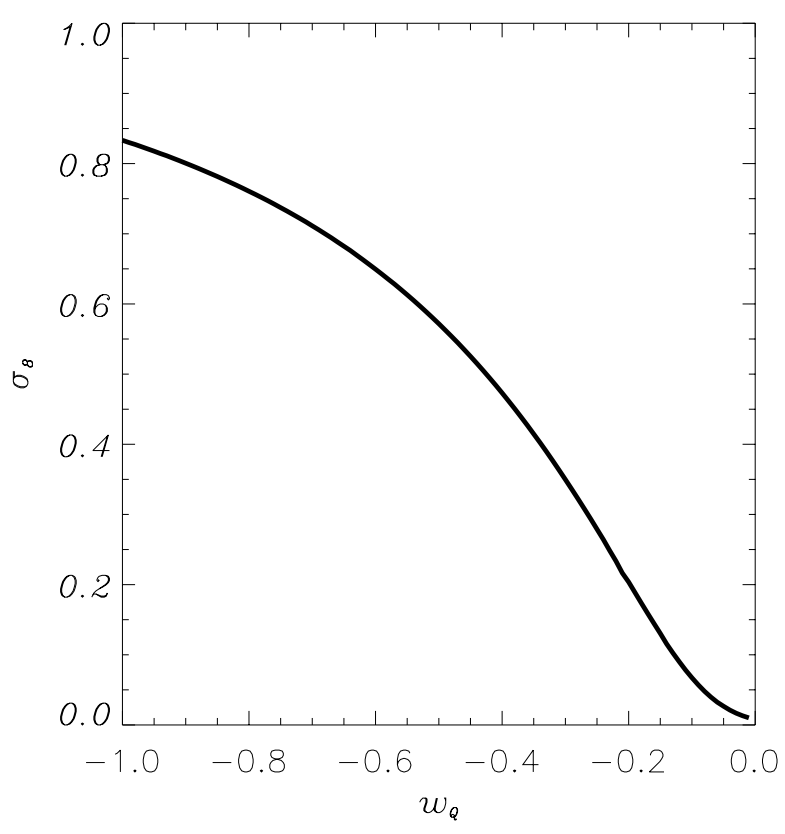

Fig. 1. The amplitude of matter fluctuations $\sigma_{8}$ as a function of a constant $w_{\mathrm{Q}}$ in cosmological models with $\Omega_{\mathrm{Q}}=0.7, \Omega_{\mathrm{b}} h^{2}=0.02$, $H_{0}=66.5, n=1$, and $\Omega_{\mathrm{tot}}=1$, with amplitude normalized to match the COBE data.

time), and $\mathcal{H} \equiv \dot{a} / a$. The only difference with the usual matterdominated case is that we do not have $\Omega_{\text {mat }}=1$ in the above equation. In the matter-dominated era, $a \propto \eta^{2}$, so that $\mathcal{H}=2 / \eta$, and

$\ddot{\delta}+\frac{2}{\eta} \dot{\delta}-\frac{6}{\eta^{2}} \Omega_{\mathrm{mat}} \delta=0$.

When $\Omega_{\text {mat }}=1$, one gets the usual result that the growing mode goes as $\delta^{+} \propto a$. If we now suppose that $\Omega_{\text {mat }}=0.3$, then the growing mode goes as $\delta^{+} \propto a^{0.466}$. If this regime lasts for several orders of magnitude in redshift, then this represents an enormous reduction in the matter power spectrum, of around $(1+\Delta z)^{1.07}$, where $\Delta z$ represent the duration of this regime. This effect will imply that $\sigma_{8}$ is drastically reduced when one goes from $w_{\mathrm{Q}}=-1$ to $w_{\mathrm{Q}}=0$. In order to illustrate this point we have computed the amplitude of matter fluctuations $\sigma_{8}$ for a family of models which have the same same cosmological parameters but the equation of state $w_{\mathrm{Q}}$ (Fig. 1). As one can see, $\sigma_{8}$ varies dramatically with $w_{\mathrm{Q}}$ and is therefore a very useful quantity to take into account in order to constrain quintessence.

\section{Methods and data}

In the following, we make use of the most recent CMB data available as well as data on other relevant cosmological quantities. With these, we examine the constraints that can be set on the amount of quintessence present in the universe. We assume Gaussian adiabatic fluctuations and a flat spatial geometry. Negligible amounts of gravitational waves are assumed. This contribution has little impact on the position of the peak, but modifies significantly the relative power between low and large $\ell$. Identically, a reionisation effect and a possible hot dark matter component are neglected. We make the assumption that $w_{\mathrm{Q}}=$ Const throughout all the epochs of interest. This assumption is unjustified in realistic quintessence models as one expects $w_{\mathrm{Q}}$ to have varied at the radiation to matter transition and to be decreasing today. However, if we want to study the influence of the quintessence field on the position of the Doppler peaks of the CMB anisotropies, then this is sufficent, and the constant $w_{\mathrm{Q}}$ has to be seen as some "average" of a dynamical $w_{\mathrm{Q}}(z)$ arising in a quintessence scenario.

\subsection{Likelihood from $C M B$}

In order to use CMB data, we first reconstruct the likelihood function of the various experiments.

We follow the technique developped in Bartlett et al. (2000), and used in Douspis et al. (2001) and Benoît et al. (2003b), by constructing a large $C_{\ell}$ power spectra database. We investigate six cosmological parameters assuming flat cosmology $\left(\Omega_{\text {tot }}=1\right)$. The density of the universe is parametrised by the baryon contribution, $\Omega_{\mathrm{b}} h^{2}$, and the dark energy, $\Omega_{\mathrm{Q}}$, for which we study various values of its pressure-energy density ratio, $w_{\mathrm{Q}}$.

The Hubble parameter, $H_{0}$, the spectral index, $n$, and the normalisation of the spectra, parametrized in this work by $\sigma_{8}$, are the remaining free parameters. Table 1 describes the corresponding gridding used for the database.

We proceed by estimating cosmological parameters from the likelihood functions reconstructed as described in Benoît et al. (2003b). We compute the value of the likelihood considering the actual band powers dataset of the COBE, BOOMERanG, DASI, MAXIMA, VSA, CBI, Archeops experiments (Tegmark 1996; Netterfield et al. 2002; Halverson et al. 2002; Lee et al. 2001; Scott et al. 2002; Pearson et al. 2002; Benoît et al. 2003a) on each model of our grid. In our approach, the best model is estimated as being that for which the likelihood is maximal $\mathcal{L}_{\max }$, while the $68 \%$ (resp. 95\%) 1-parameter interval corresponds to $-2 \ln \left(\mathcal{L} / \mathcal{L}_{\max }\right) \in[0,1]$ (resp. $\left.-2 \ln \left(\mathcal{L} / \mathcal{L}_{\text {max }}\right) \in[0,4]\right)$ and the $68 \%$ (resp. 95\%) 2 parameters interval corresponds to $-2 \ln \left(\mathcal{L} / \mathcal{L}_{\max }\right) \in[0,2.3]$ (resp. $\left.-2 \ln \left(\mathcal{L} / \mathcal{L}_{\max }\right) \in[0,6.18]\right)$. The likelihoods shown in the following are already marginalized (by maximisation) over the calibration uncertainties and the amplitude. The results are presented as 2D contour plots, showing in shades of blue the regions where the likelihood function for a combination of any two parameters drops to the levels corresponding to $68 \%, 95 \%$, and $99 \%$ confidence regions. They would correspond to 1,2 , $3 \sigma$, respectively if the likelihood function were Gaussian. Dashed red contours mark the limits to be projected if confidence intervals are sought for any one of the parameters. To calculate either 1 or $2 \mathrm{D}$ confidence intervals, the likelihood function is maximized over the remaining parameters.

\subsection{Other data}

In order to include other data of cosmological relevance, the corresponding additional likelihood have to be evaluated. 
Table 1. Grid of explored cosmological parameters; for $H_{0}$ we adopt a log-periodic binning, $H_{0}(i+1)=H_{0}(i) \times 1.15$.

\begin{tabular}{ccccccc}
\hline \hline & $w_{\mathrm{Q}}$ & $\Omega_{\mathrm{Q}}$ & $100 \Omega_{\mathrm{b}} h^{2}$ & $H_{0}$ & $n$ & $\sigma_{8}$ \\
\hline Min. & -1.0 & 0.0 & 0.915 & 25 & 0.750 & 0.1 \\
Max. & -0.1 & 1.0 & 3.47 & 101 & 1.25 & 1.3 \\
Step & 0.1 & 0.1 & 0.366 & $\times 1.15$ & 0.015 & 0.022 \\
\hline
\end{tabular}

An interesting useful additional constraint to add is that obtained from the amplitude of matter fluctuations on small scales: present day clusters data allow one to constrain $\sigma_{\mathrm{c}}$, related to $\sigma_{8}$ to a high accuracy of the order of $5 \%$ (Blanchard et al. 2000). A similar constraint can be obtained from weak lensing measurements (Bacon et al. 2000; van Waerbeke et al. 2002). However, significant differences among similar analyses have appeared in recent works based on clusters as well as on weak lensing measurements (Jarvis et al. 2003; Brown et al. 2003; Hamana et al. 2002). We have therefore chosen to use two recent constraints, the differences of which will allow us to investigate a realistic range of systematic uncertainties. First we consider a constraint leading to high values of $\sigma_{8}$ (high $\sigma_{8}$ hereafter), in agreement with Pierpaoli et al. (2001): $\sigma_{8} \Omega_{\text {mat }}^{0.6}=0.5 \pm 10 \%$ ( $68 \%$ C.L.). Then, new estimations from Seljak et al. (2002), Viana et al. (2002), Reiprich et al. (2002), Hoekstra et al. (2002) leading to lower values, are considered by taking $\sigma_{8} \Omega_{\text {mat }}^{0.38}=0.43 \pm 10 \%$ (68\% C.L.), hereafter low $\sigma_{8}$. The latter error bar estimates encompass the three above low normalization measurements of $\sigma_{8}$.

We also use the recent determination of the Hubble parameter from the Hubble Space Telescope (HST) Key Project (Freedman et al. 2001): $H_{0}=72 \pm 8$ (68\% C.L.), assuming Gaussian uncertainty.

For the distant supernovae constraints we compute the likelihood as follows. The magnitude-redshift relation of the supernovae is given by the following relation

$M=\mathcal{M}+5 \log _{10} \mathcal{D}_{L}\left(z, w_{\mathrm{Q}}, \Omega_{\mathrm{Q}}\right)$,

where $M$ is the observed magnitude and $\mathcal{D}_{L}$ and $\mathcal{M}$ are respectively the "Hubble-parameter-free" luminosity-distance and the "Hubble-parameter-free" absolute magnitude at the maximum of the supernova. $\mathcal{D}_{L}$ is a function of redshift of the supernova, $z$, and of the cosmological parameters $w_{\mathrm{Q}}$ and $\Omega_{\mathrm{Q}}$. In the case of a flat universe with $w_{\mathrm{Q}}$ as the pressure to energy density ratio of the quintessence field, the $\mathcal{D}_{L}$ function is given by

$$
\begin{aligned}
\mathcal{D}_{L}\left(z, w_{\mathrm{Q}}, \Omega_{\mathrm{Q}}\right) & = \\
(1+z) \int_{0}^{z} & \frac{\mathrm{d} u}{\sqrt{\left(1-\Omega_{\mathrm{Q}}\right)(1+u)^{3}+\Omega_{\mathrm{Q}}(1+u)^{3\left(1+w_{\mathrm{Q}}\right)}}} .
\end{aligned}
$$

With the sample of supernovae from Perlmutter et al. (1999), we construct the likelihood $\mathcal{L}_{\mathrm{SN}}$ with the following procedure:

$\mathcal{L}_{\mathrm{SN}}=C \exp \left[\sum_{i}\left(-\frac{\left(M_{i}^{\mathrm{obs}}-M_{i}\left(\mathcal{M}, w_{\mathrm{Q}}, \Omega_{\mathrm{Q}}\right)\right)^{2}}{2 \sigma_{i}^{2}}\right)\right]$,

where $C$ is an arbitrary constant. We take for measured magnitude $M^{\text {obs }}$ and uncertainty $\sigma$ the $B$-band peak magnitude and
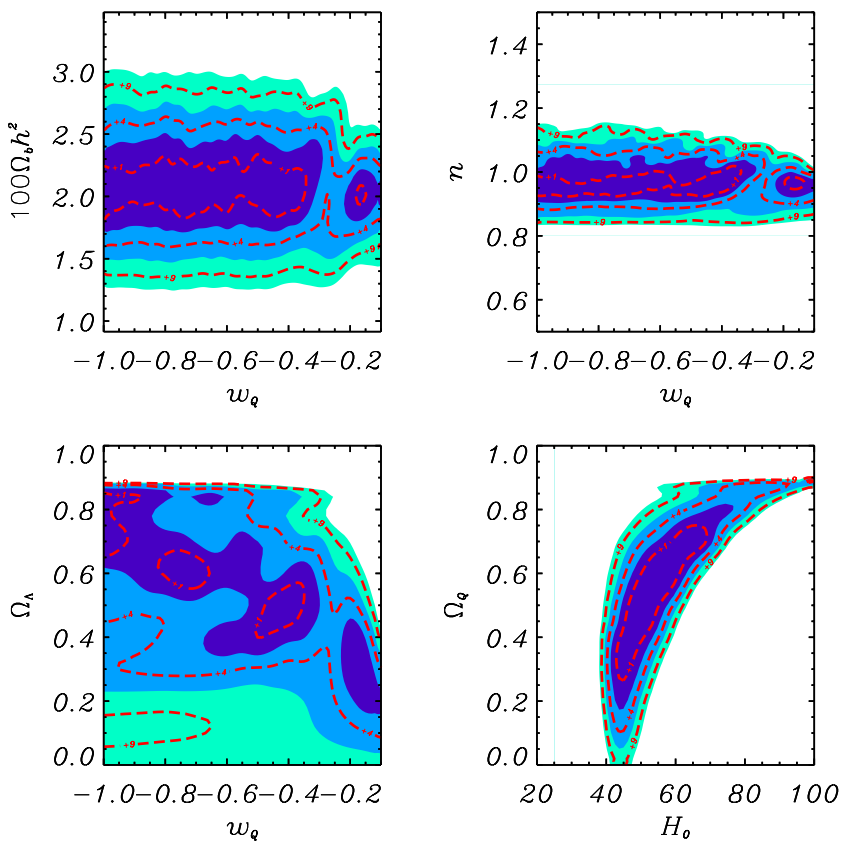

Fig. 2. Present CMB dataset likelihood contours in the quintessence paradigm. The sharpness of contours at $\Omega_{\mathrm{Q}}=0.9$ is due to grid effect.

the total uncertainty of the supernovae from Perlmutter et al. (1999). At each point of the two parameters grid, $w_{\mathrm{Q}}$ and $\Omega_{\mathrm{Q}}$ is associated a likelihood value obtained by maximising it over the absolute magnitude $\mathcal{M}$.

\section{Results}

\subsection{CMB alone}

Constraints given by the $\mathrm{CMB}$ on some of our investigated parameters are shown in Fig. 2. Considering only CMB constraints leads to degeneracies between parameters. Figure 2 shows the case of two parameters, $n$ and $\Omega_{\mathrm{b}} h^{2}$, which are not affected by the assumed equation of state of the dark energy. Their prefered values and error bars are $n=0.95 \pm 0.05$ and $\Omega_{\mathrm{b}} h^{2}=0.021 \pm 0.003$ (68\% C.L.). Using CMB alone leaves the 2-parameter space $\left(\Omega_{\mathrm{Q}}, w_{\mathrm{Q}}\right)$ almost unconstrained. Finally, 2D diagrams $\Omega_{\mathrm{Q}}$ vs. $H_{0}$ or $w_{\mathrm{Q}}$ vs. $H_{0}$ show a significant level of correlation, but with degeneracies. This is illustrated by the plot $\Omega_{\mathrm{Q}}$ vs. $H_{0}$ shown in Fig. 2. In our analysis, we found that with the improvement of CMB data obtained by the addition of Archeops band powers reduces appreciably the contours of constraints on the quintessence parameters as well as on cosmological parameters because the position and the amplitude of the first acoustic peak are better determined, but still does not allow to break the degeneracies.

\subsection{Adding non CMB priors}

As cluster abundance observations lead to a strong constraint on the normalization of the matter power spectrum, and $\sigma_{8}$ is rather sensitive to the changing the equation of state, it is natural to expect that this constraint in combination with constraints from $C_{\ell}$ will lead to tight constraints on quintessence 

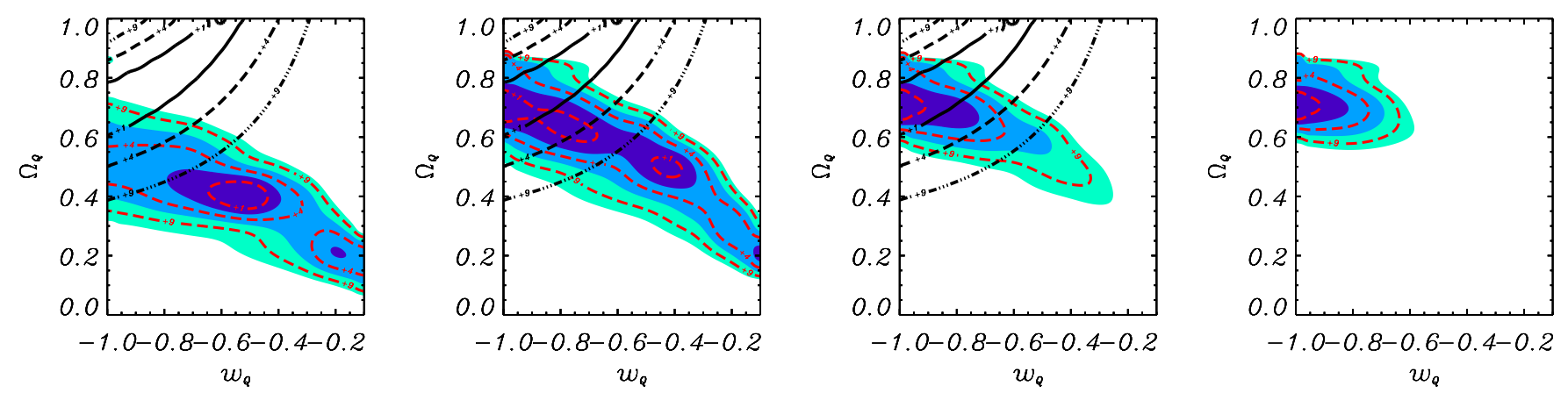

Fig. 3. Likelihood contours with combination of CMB and priors. From left to right, panels show (i) colored contours corresponding to the combination $\mathrm{CMB}+\sigma_{8}$ (high), wiht overplotted SN contours; (ii) colored contours corresponding to the combination $\mathrm{CMB}+\sigma_{8}$ (low), with overplotted SN contours; (iii) colored contours corresponding to the combination CMB $+\sigma_{8}$ (low) + HST, with overplotted SN contours; (iv) colored contours corresponding to the combination $\mathrm{CMB}+\sigma_{8}(\mathrm{low})+\mathrm{HST}+\mathrm{SN}$.

scenarios. Figure 3 (left panels) shows the combination of CMB data with the two $\sigma_{\mathrm{c}}$ priors described previously. As one can see, constraints on the amplitude of matter fluctuations on small scales has the potential to break the degeneracies between $\Omega_{\mathrm{Q}}$ and $w_{\mathrm{Q}}$. Only a band-shaped region of the plane is not excluded. Furthermore, combinations with different priors lead to different likelihood contours, due to the strong effect of the equation of state on $\sigma_{8}$ emphasized in Sect. 2. More specifically, we find that the CMB, combined with the high normalization leads to a prefered region which is marginally consistent with the constraints given by high redshift supernovae (overplotted black lines in Fig. 3). The "concordance model" $\left(\Omega_{\mathrm{Q}}=0.7, w_{\mathrm{Q}}=-1\right)$ lies on the $99 \%$ C.L. contours, and defines the two dataset as inconsistent. The best model appears then to have $\Omega_{\mathrm{Q}}=0.4, w_{\mathrm{Q}}=-0.5$, which is itself outside the $99 \%$ confidence region of the supernovae constraints. The combination of CMB with low normalization leads, on the other hand, to likelihood contours in agreement with those of supernovae and HST key project determination of $\mathrm{H}_{0}$.

Due to the form of the joint $\mathrm{CMB}+\sigma_{8}$ (high or low) contours, a combination with the HST constraints is expected to give stronger constraints on both $\Omega_{\mathrm{Q}}$ and $w_{\mathrm{Q}}$. The corresponding likelihood contours of Fig. 3 (middle right panel) show that quintessence is not prefered over classical cosmological constant even if the degeneracy is not totally broken: $\Omega_{\mathrm{Q}}=0.70_{-0.12}^{+0.16}, w_{\mathrm{Q}}=-1^{+0.4}$ (95\% C.L.).

In order to break the degeneracy, it is clearly necessary to consider the additional information on the angular distance coming from distant supernovae. Considering a flat cosmology, the information on the luminosity of the supernovae can be expressed in term of constraints on the dark energy density and equation of state. Prefered values are consistent with a cosmological constant, and the likelihood contours are almost perpendicular to those of CMB, as shown in Fig. 3. Combining all the priors finally allows to put strong constraints on both quintessence parameters (Fig. 3, rightmost panel): $\Omega_{\mathrm{Q}}=0.70_{-0.17}^{+0.10}, w_{\mathrm{Q}}=-1^{+0.25}$ (95\% C.L.) and finally breaks the $\left(H_{0}, \Omega_{\mathrm{Q}}\right)$ degeneracy, see Fig. 4 .

As a main result, it appears that the classical $\Lambda C D M$ scenario is still prefered and given the priors we used there is no need for quintessence to reproduce the present data, although quintessence models with low $w_{\mathrm{Q}}$ are still viable, and that good fits to the data can also be found for models with $w_{\mathrm{Q}}<-1$ (Melchiorri et al. 2002) ${ }^{1}$. However, those correspond to rather unusual quintessence models.

Going back to typical quintessence models, finding only low values of $w_{\mathrm{Q}}$ raises some interesting points. If we consider a pure inverse power law potential, having $w_{\mathrm{Q}}<-0.6$ when $\Omega_{\mathrm{Q}}=0.7$ implies a low value of the exponent $\alpha$ (if the slope of the potential is too steep, the field does not stop rapidly when it starts dominating, and $w_{\mathrm{Q}}$ is too large). This translates into an annoyingly low value of the energy scale $M$ arising in the potential, since one has $M \sim M_{\mathrm{Pl}}\left(\rho_{\mathrm{c}} / M_{\mathrm{Pl}}^{4}\right)^{1 /(4+\alpha)}$ (Brax et al. 2000). For example, in order to have $M>10^{3} \mathrm{TeV}$, one needs $\alpha \geq 6$, whereas data favor $w_{\mathrm{Q}}<-0.6$, which corresponds to $\alpha \lesssim 3$, or $M \lesssim 20 \mathrm{GeV}$. There are of course several quintessence models which predict a lower $w_{\mathrm{Q}}$. For example, the SUGRA potential found by Brax \& Martin (1999), is given by $V=M^{4+\alpha} / \phi^{\alpha} \times \exp \left(\frac{1}{2} \phi^{2} / M_{\mathrm{Pl}}^{2}\right)$. The exponential correction comes from supergravity considerations and insures that the potential has a local minimum which is almost reached by the field today. For example, for $\Omega_{\mathrm{Q}}=0.7$, one has $w_{\mathrm{Q}}=-0.8$, almost independently of $\alpha$. This illustrates the fact that it is still difficult to distinguish some quintessence models from a cosmological constant with the present state of available data. On the other hand, the data already allow one to exclude a large number of quintessence models, among which the simplest.

\section{Conclusion}

We have studied the constraints that can be obtained on cosmological parameters within the quintessence paradigm by using various combinations of observational data. For simplicity, only models with constant $w_{\mathrm{Q}}$ were examined: given the accuracy of existing data, we believe this to be a sufficient approximation. For similar reasons, we neglected possible reionization or non-zero gravitational wave contributions. This approach has the advantage to helping us to understand whether quintessence models are favored or not over a classical

\footnotetext{
1 During the preparation of this work, another group submitted a related paper to the archive (Melchiorri et al. 2002). Although the analysis is not exactly the same, their conclusions are similar.
} 

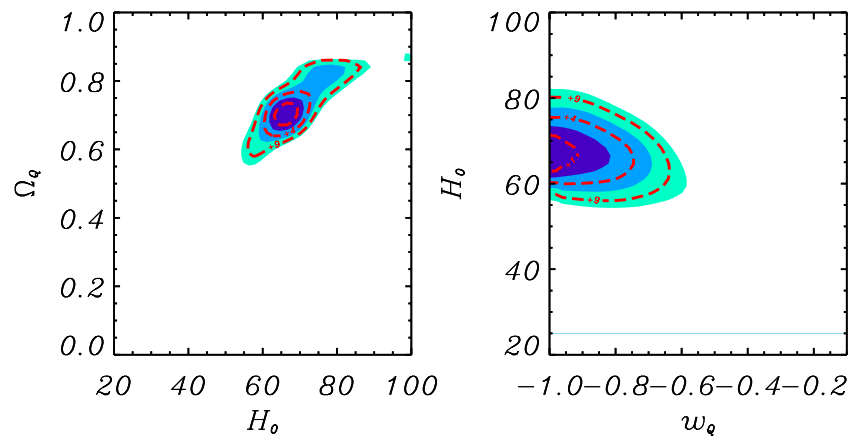

Fig. 4. Likelihood contours with $\mathrm{CMB}+$ all priors.

cosmological constant, while constraints on more specific scenarios have to be investigated directly one by one (Douspis et al. 2003). Our analysis method has been to investigate contours in 2D parameters space. Such an approach allows one to examine possible degeneracies among parameters which are not easy to identify when constraints are formulated in term of a single parameter. For instance, we found that, despite the high precision data obtained by Archeops, CMB data alone does not require the existence of a non-zero contribution of quintessence, because of the degeneracy with the Hubble parameter: in practice CMB data leaves a large fraction of the $\left(\Omega_{\mathrm{Q}}, w_{\mathrm{Q}}\right)$ plane unconstrained, while only a restricted region of the $\left(\Omega_{\mathrm{Q}}, H_{0}\right)$ plane is allowed. On the contrary, we found that almost no correlation exists with the baryonic content $\Omega_{\mathrm{b}}$ nor the primordial index $n$. In order to restrict the parameter space of allowed models, we have applied several different constraints. Interestingly, we found that the amplitude of the dark matter fluctuations, as measured by clusters abundance or large scale weak lensing data can potentially help to break existing degeneracies, although existing uncertainties, mainly systematics in nature, do not allow for a firm conclusion as yet. Clearly this will be an important check of consistency in the future. We have then added constraints from supernovae data as well as HST estimation of the Hubble parameter in order to break existing degeneracies. This allows us to infer very tight constraints on the possible range of equation of state of the dark energy. Probably the most remarkable result is that no preference for quintessence emerges from existing CMB data although accurate measurement of the amplitude of matter fluctuations on scale of $8 h^{-1}$ Mpc may change this picture.

Acknowledgements. The authors acknowledge the use of the CAMB code (Lewis et al. 2000). M.D. is on a CMBNet fellowship and acknowledges Oxford Astrophysics group computational facilities and the Archeops collaboration. Y.Z. acknowledges support from the CNRS. This work has greatly benefitted from discussions inside the Archeops collaboration

\section{References}

Bacon, D. J., Refregier, A. R., \& Ellis, R. S. 2000, MNRAS, 318, 625

Bartlett, J. G., Douspis, M., Blanchard, A., \& Le Dour, M. 2000, A\&AS, 146, 507

Benabed, K., \& Bernardeau, F. 2001, Phys. Rev. D, 64, 083501

Benoît, A., Ade, P., Amblard, A., et al. (Archeops collaboration) 2002, Astropart. Phys., 17, 101

Benoît, A., Ade, P., Amblard, A., et al. (Archeops collaboration) 2003a, A\&A, 399, L19

Benoit, A., Ade, P., Amblard, A., et al. (Archeops collaboration) 2003b, A\&A, 399, L25

Binétruy, P. 1999, Phys. Rev. D, 60, 063502

Binétruy, P. 2000, Int. J. Theor. Phys. 39, 1852

Blanchard, A., Sadat, R., Bartlett, J., \& Le Dour, M. 2000, A\&A, 362, 809

Brax, P., \& Martin, J. 1999, Phys. Lett. B468 40

Brax, P., Martin, J., \& Riazuelo, A. 2000, Phys. Rev. D, 62, 103505

Brown, M. L., Taylor, A. N., Bacon, D. J., et al. 2003, MNRAS, 341, 100

Caldwell, R. R., Dave, R., \& Steinhardt, P. 1998, Phys. Rev. Lett., 88, 1582

Douspis, M., Blanchard, A., Sadat, R., Bartlett, J. G., \& Le Dour, M. 2001, A\&A, 379, 1

Douspis, M., Riazuelo, A., Blanchard, A., \& Zolnierowski, Y. 2003, in preparation

Freedman, W. L., Madore, B. F., Gibson, B. K., et al. 2001, ApJ, 553, 47

Halverson, N. W., Leitch, E. M., Pryke, C., et al. 2002, MNRAS, 568, 38

Hamana, T., Miyazaki, S., Shimasaku, K., et al. 2002, ApJ, submitted [astro-ph/0210450]

Hoekstra, H., Yee, H., \& Gladders, M. 2002, ApJ, 577, 595

Jarvis, M., Bernstein, G., Jain, B., et al. 2003, AJ, 125, 1014

Lee, A. T., Ade, P., Balbi, A., et al. 2001, ApJ, 561, L1

Lewis, A., Challinor, A., \& Lasenby, A. 2000, ApJ, 538, 473

Melchiorri, A., Mersini, L., Ödman, C. J., \& Trodden, M. 2002 [astro-ph/0211522]

Netterfield, C. B., Ade, P. A. R., Bock, J. J., et al. 2002, ApJ, 571, 604

Pearson, T. J., Mason, B. S., Readhead, A. C. S., et al. 2002, ApJ, accepted [astro-ph/0205388]

Perlmutter, S., Aldering, G., Goldhaber, G., et al. 1999, ApJ, 517, 565; see also http://www-supernova. lbl.gov

Pierpaoli, E., Scott, D., \& White, M. 2001, MNRAS, 325, 77

Ratra, B., \& Peebles, P. J. E. 1988, Phys. Rev. D, 37, 3406

Reiprich, T. H., \& Böhringer, H. 2002, ApJ, 567, 716

Scott, P. F., Carreira, P., Cleary, K., et al. 2002, MNRAS, in press [astro-ph/0205380]

Seljak, U. 2002, MNRAS, 337, 769

Steinhardt, P., Wang, L., \& Zlatev, I. 1999, Phys. Rev. D, 59, 123504

Tegmark, M. 1996, ApJ, 464, L35

Van Waerbeke, L., Mellier, Y., Pelló, R., et al. 2002, A\&A, 393, 369

Viana, P. T. P., Nichol, R. C., \& Liddle, A. R. 2002, ApJ, 569, L75

Wetterich, C. 1988, Nucl. Phys. B302, 668 